\title{
Preface: Structure and Evolution
}

In the twenty-first century, we highly depend on data and are part of the information society, where huge function platforms have been established by online social networks. We state our viewpoints, make friends, and interact on Twitter, Facebook, LinkedIn, Sina Microblog, WeChat, and other social networks. Lots of information generated each day are conveniently available to people. Online social activities are changing human behavior models and social formation, and social network data is becoming the most mature big data. By using the technique of big data behind every online social networks, we can hope that people's understanding on user behaviors and social phenomenon may reach an unprecedented depth.

Online social network analysis relates to computing science, sociology, management, psychology, and many other subject areas. As a chief scientist of the Major State Basic Research Development Program of China (973 Program), that is, "Fundamental Research of Social Network Analysis and Network Information Diffusion," and during my research on online social networks, I felt that this field lacked a treatise, which systematically elaborates the concepts, theories, and techniques of online social network analysis from a multidisciplinary aspect. Hence, I formed a team consisting of 973 Program, including National University of Defense Technology, Shanghai Jiao Tong University, Hefei University of Technology, Beijing University of Post \& Telecommunications, Institute of Computing Technology, CAS, Peking University, University of Science and Technology Beijing, Institute of Information Engineering, CAS, and Harbin Institute of Technology. This book is a result of the research findings of these teams and a systematic review of relevant theories and techniques globally, so as to provide theoretical, systematic, and instrumental research guides for relevant researchers.

The three core factors for the analysis of online social networks are "structure and evolution," "groups and interaction," and "information and diffusion."

Volume 1 of this book consists of four chapters. Chapter 1 provides introduction for the book, followed by Chapters 2-4 that focus on the first core factor, namely, "structure and evolution." Chapter 2 is about the analysis and modeling of social network structure characteristics, Chapter 3 is about the detection techniques and approaches for virtual communities, and Chapter 4 discusses about the evolution and analysis of virtual communities.

The following experts and scholars who participated in the data collection, content arrangement, and achievement contribution of this volume are sincerely appreciated: Zhaoyun Ding, Xiaomeng Wang, Bin Wang, Yezheng Liu, Xiaodong Liu, Shenghong Li, Aiping Li, Lei Li, Shiyu Du, Peng Wu, Xiuzhen Chen, Wei Chen, Yang Yang, Lumin Zhang, Peng Shi, and Yuanchun Jiang.

Thanks to Associate Professor Shudong Li for the careful coordination and arrangement for writing this volume, and also to Weihong Han and Shuqiang Yang for reviewing and proofreading. 
